\title{
Migration with Local Public Goods and the Gains from Changing Places
}

\author{
Peter J. HAMmond \\ Department of Economics, University of Warwick, Coventry CV4 7AL, U.K. \\ p.j.hammond@warwick.ac.edu \\ JAUMe SEMPERE \\ C.E.E., El Colegio de México, Camino al Ajusco 20, \\ Pedregal de Santa Teresa, 10740 México D.F., MÉxico \\ jsempe@colmex.mx
}

Preliminary version: August 2000; latest version: 23rd July 2007.

\begin{abstract}
Without public goods and under fairly standard assumptions, in Hammond and Sempere (2006) we show that freeing migration enhances the potential Pareto gains from free trade. Here we present a generalization allowing local public goods subject to congestion. Unlike the standard literature on fiscal externalities, our result relies on fixing both local public goods and congestion levels at their status quo values. This allows constrained efficient and potentially Pareto improving population exchanges regulated only through appropriate residence charges, which can be regarded as Pigouvian congestion taxes.
\end{abstract}

JEL Classification: F22, F13, D61, C62.

Keywords: Migration, gains from trade, general equilibrium, non-convexities, indivisibilities, local public goods, congestion. 


\section{Introduction}

Standard first-best results on the gains from trade require demonstrating the existence of a competitive equilibrium with lump-sum redistribution arranged to ensure that everybody gains — or at least nobody loses relative to the status quo. When there are no public goods, in Hammond and Sempere (2006) we present similar results on the gains from migration. There the main obstacle to proving a suitable existence theorem was the obvious non-convexity that arises because no potential migrant can be in more than one place at the same time. Nevertheless, in a continuum economy with appropriately dispersed agent characteristics, standard assumptions ensuring gains from trade also ensure gains from migration.

A second apparent obstacle to achieving a potential Pareto improvement from free migration arises from public goods and externalities, which are ignored in most of the existing literature on gains from trade. Yet neglecting local public goods subject to congestion seems especially damaging. ${ }^{1}$ Because the cost of providing such goods is obviously affected by migration, no simple extension of our earlier results is possible. Nevertheless, we argue in this paper that potential gains from trade and from appropriate migration remain possible, provided that both the provision of public goods and the congestion levels affecting those goods are frozen at their status quo lev-

\footnotetext{
${ }^{1}$ We recall the important distinction between pure public goods and public goods subject to congestion. Examples of pure public goods include broadcast radio or television, streetlighting, etc., whose cost of provision is not directly related to population, so is unaffected by migration or congestion. If all public goods were of this kind, routine modifications of our earlier results imply that Pareto gains from free migration are possible in an economy where the supplies of public goods in each nation or locality are frozen at their status quo levels.
} 
els. Of course, such results do not exclude additional potential Pareto gains from appropriate changes in public good supply and/or congestion levels. Our point is that such changes are not needed in order to allow potential Pareto gains to emerge from a combination of free trade and free exchange of population.

The paper is organized as follows. First, sections 2-3 describe the basic model. Section 4 introduces congestion costs, as well as appropriate residence charges that can mediate efficient population exchange. Section 5 defines "sagacious" wealth distribution rules. Section 6 demonstrates existence of a (suitably constrained) compensated equilibrium. Section 7 introduces additional assumptions required to ensure that at least one commodity price is positive in that compensated equilibrium. Finally, using appropriate dispersion and convexity assumptions, Section 8 demonstrates the main result that there is an appropriate conditional competitive equilibrium. This proves that potential Pareto gains from adding free migration to free trade are possible even in the presence of national or local public goods subject to congestion. Section 9 provides a brief concluding discussion.

\section{Notation, Model and Basic Assumptions}

\subsection{Nations, Consumers and Personal Histories}

Suppose the world consists of a finite set $K$ of different countries - or, more generally, different physical locations - indexed by $k$. To allow time for migration as well as uncertainty, consider an intertemporal Arrow-Debreu economy in which $D$ is the finite set of relevant date-event pairs, with a tree structure starting at $d=0$. 
Suppose there is a fixed continuum of consumers $I$ indexed by $i$, with $\mathcal{I}$ as a $\sigma$-field of measurable subsets. Also, let $\nu$ be the appropriate measure, so that $(I, \mathcal{I}, \nu)$ is the atomless measure space of consumers. ${ }^{2}$

Each individual consumer $i \in I$ is assumed to have a migration plan in the form of a mapping $k^{i D}: D \rightarrow K$. Thus, $k^{i}(d)$ indicates the nation in which consumer $i$ plans to reside and function as an economic agent at each date-event pair $d \in D$. Obviously, the set of all possible migration plans is the (finite) Cartesian product set $K^{D}$. At $d=0$, history determines $k^{i}(0)$ as the nation which the consumer inhabits as the economy starts.

Our model of how migration affects congestion will recognize that different kinds of consumer may place different burdens on the public sectors of the nations or localities they inhabit. So we extend the description of each consumer $i \in I$ to include, for each date-event $d \in D$, an index $e^{i}(d) \in E$ of all relevant demographic or other household characteristics affecting the costs of providing local public goods. Examples of such characteristics are a particular household's entitlements in different date-event pairs for publicly provided education, health services, welfare payments, pensions, etc. Essentially, in the static model of Conley and Wooders $(1996,1997)$, each $e \in E$ is a "crowding type." We assume that the range $E$ of possible characteristics is a finite set, and let $e^{i D}=\left\langle e^{i}(d)\right\rangle_{d \in D} \in E^{D}$ denote consumer $i$ 's characteristic history.

Each consumer $i$ 's migration plan and characteristic history are jointly represented by the pair $\left(k^{i D}, e^{i D}\right)$ which belongs to the Cartesian product domain $K^{D} \times E^{D}$ of personal histories. For the remainder of the paper it will be convenient to represent each consumer $i$ 's chosen personal history by

\footnotetext{
${ }^{2}$ In Hammond and Sempere (2006) we discuss why it might be better not to let $I$ be the unit interval, and how to construct the appropriate non-atomic probability measure $\nu$.
} 
indicator variables defined by

$$
m_{k}^{i}(e, d):= \begin{cases}1 & \text { if } k^{i}(d)=k \text { and } e^{i}(d)=e \\ 0 & \text { otherwise }\end{cases}
$$

In this way the set $M$ of possible personal histories $m^{i}$ becomes a subset of

$\mathbb{R}^{E D K}$, but with each member having exactly one component $m_{k}^{i}(e, d)$ equal to 1 for each $d \in D$, with all others equal to 0 . In particular, each $m^{i}$ has exactly \#D non-zero components.

\subsection{Demographic Histories as Congestion Profiles}

The proportion of the world's inhabitants living in country $k$ and having characteristic $e$ at date-event $d$ is given by

$$
\mu_{k}(e, d):=\nu\left(\left\{i \in I \mid m_{k}^{i}(e, d)=1\right\}\right) .
$$

So at any date-event $d \in D$, the statistical distribution of consumers in country $k$ having different characteristics $e \in E$ is specified by the vector of proportions $\mu_{k}^{E}(d) \in \mathbb{R}_{+}^{E}$. Let $\mu_{k}^{E D}:=\left\langle\mu_{k}^{E}(d)\right\rangle_{d \in D} \in \mathbb{R}_{+}^{E D}$ denote the "national demographic history." Let $\mu^{E D K}:=\left\langle\mu_{k}^{E D}\right\rangle_{k \in K}$ denote the corresponding "world demographic history," which we usually just call a congestion profile. Note that $\mu^{E D K}=\int_{I} m^{i} d \nu$.

\subsection{Private Goods}

Assume that there is a finite set $G(d)$ of dated contingent private commodities in each date-event $d \in D$. Suppose this set is partioned into pairwise disjoint components $T(d)$ and $N_{k}(d)(k \in K)$, where $N_{k}(d)$ is the set of goods in date-event $d$ specific to country $k$ that are not traded internationally, and $T(d)$ is the set of internationally traded goods. We assume that each set 
$N_{k}(d)$ includes all relevant kinds of labour, since labour is not traded directly across borders. Rather, migrants move across borders to supply labour in other nations. Then the relevant set of dated contingent commodities is $G:=\cup_{d \in D} G(d)$ and the finite-dimensional commodity space is $\mathbb{R}^{G}$.

Let $G_{k}(d):=T(d) \cup N_{k}(d)$ denote the subset of goods that can be traded in nation $k$ at date-event $d$. Also, let $G_{k}:=\cup_{d \in D} G_{k}(d)$ denote the subset of all goods that can be traded in nation $k$; these will be the goods which can appear as inputs and/or outputs in production activities within nation $k$. Finally, let $G(m):=\cup_{d \in D} G_{k(d)}(d)$ denote the subset that can be traded by any consumer with the personal history $m=\left(k^{D}, e^{D}\right)$.

\subsection{Fixed Public Goods and Congestion Profile}

A Pareto efficient allocation of private and public goods also requires an efficient allocation of congestion, with the appropriate level of the profile $\mu^{E D K}$. However, there is no need here to assume that any such efficient allocation of total congestion occurs.

Instead, we consider what happens when public good provision is fixed at its status quo value, as is the congestion profile $\mu^{E D K}$. In particular, individuals are allowed to migrate and to change their congestion characteristics. But in the equilibrium we look for there must be other individuals choosing to make offsetting changes whose overall effect is that the congestion profile $\mu^{E D K}$ remains at its status quo level $\bar{\mu}^{E D K}$. That is, individuals are restricted to exchanging places in equilibrium. Even so, in addition to the usual gains from trade, there can still be gains from migration in the form of appropriate population exchanges between different countries, allowing each household to go wherever it wants taking into account its preferences, 
its opportunities to earn labour income, and also what residence charges it is expected to pay.

\section{Consumers' Characteristics}

\subsection{Feasible Sets}

Each consumer $i \in I$ will be modelled as choosing some pair $\left(x^{i}, m^{i}\right)$ that consists of a net trade vector $x^{i} \in \mathbb{R}^{G}$ and a personal history $m^{i} \in \mathbb{R}^{E D K}$.

Different personal histories obviously incur different costs. Also, different vectors of public goods in different localities allow different quantities of private goods to be consumed, depending on the consumer's own personal history, including migration decisions. For these reasons, we assume that each consumer $i$ 's net trade vector $x^{i} \in \mathbb{R}^{G}$ is restricted to a conditional feasible set $X^{i}\left(m^{i}\right)$ that is compatible with the chosen personal history $m^{i} \in$ $M$ and the frozen public good vector. Formally:

(A.1) For every personal history $m^{i} \in M$, each consumer $i \in I$ has a (possibly empty) closed conditional feasible set $X^{i}\left(m^{i}\right)$ satisfying $x_{g}^{i}=0$ for all $g \notin G\left(m^{i}\right)$. Also, the free disposal of $i$ 's tradable commodities condition holds - requiring that, whenever $x^{i} \in X^{i}\left(m^{i}\right)$ and $\tilde{x}^{i} \geqq x^{i}$ with $\tilde{x}_{g}^{i}=0$ for

all $g \notin G\left(m^{i}\right)$, then $\tilde{x}^{i} \in X^{i}\left(m^{i}\right)$. In addition, each consumer $i \in I$ has a non-empty overall conditional feasible set defined by

$$
X^{i}:=\left\{\left(x^{i}, m^{i}\right) \in \mathbb{R}^{G} \times K^{D} \mid x^{i} \in X^{i}\left(m^{i}\right)\right\} .
$$

\subsection{Preferences}

Given fixed public good vectors in each locality, consumers will be allowed to have preferences over combinations of personal histories and net trade vec- 
tors. In our continuum economy, there is no need to assume that preferences are convex. So our next assumption is:

(A.2) Each consumer $i \in I$ has a weak preference relation $\succsim^{i}$ defined on $X^{i}$ that is reflexive, complete, transitive, continuous, as well as weakly monotonic in $i$ 's tradable commodities in the sense that, whenever $x^{i} \in X^{i}\left(m^{i}\right)$ and $\tilde{x}^{i} \geqq x^{i}$ with $\tilde{x}_{g}^{i}=0$ for all $g \notin G\left(m^{i}\right)$ and $\tilde{x}_{g}^{i}>x_{g}^{i}$ for all $g \in G\left(m^{i}\right)$, then $\left(\tilde{x}^{i}, m^{i}\right) \succ^{i}\left(x^{i}, m^{i}\right)$, where $\succ^{i}$ denotes the corresponding strict preference relation.

Note that $\left(x^{i}, m^{i}\right) \in X^{i} \Longleftrightarrow\left(x^{i}, m^{i}\right) \succsim^{i}\left(x^{i}, m^{i}\right)$ because $\succsim^{i}$ is com-

plete. So each consumer $i$ 's feasible set $X^{i}$ and preference relation $\succsim^{i}$ are characterized completely by the graph

$$
\Gamma^{i}:=\left\{\left(x^{i}, m^{i}, \tilde{x}^{i}, \tilde{m}^{i}\right) \in \mathbb{R}^{G} \times M \times \mathbb{R}^{G} \times M \mid\left(x^{i}, m^{i}\right) \succsim^{i}\left(\tilde{x}^{i}, \tilde{m}^{i}\right)\right\}
$$

of $\succsim^{i}$. By continuity, this is a closed subset of $\mathbb{R}^{G} \times M \times \mathbb{R}^{G} \times M$.

\subsection{Characteristics}

As has become standard since the work of Hildenbrand (1974), we assume:

(A.3) The consumer characteristic space $\Theta$ of feasible sets $X$ and of preference relations $\succsim$, as represented by their closed graphs $\Gamma$, is endowed with the topology of closed convergence and the associated Borel $\sigma$-field $\mathcal{B}$. Moreover, the mapping $i \mapsto \Gamma^{i}$ from $I$ to $\mathbb{R}^{G} \times M \times \mathbb{R}^{G} \times M$ is measurable w.r.t. the respective $\sigma$-fields $\mathcal{I}$ and $\mathcal{B}$. 


\section{Producers, Feasible Allocations and Profits}

\subsection{National and International Production}

In this paper there is no need to pay attention to individual producers or their net output vectors. Instead, all our analysis will involve the aggregate net output vector $y_{k}$ and aggregate production set of each nation $k \in K$. We assume:

(A.4) Each nation $k \in K$, given its fixed public environment and status quo congestion levels, has a closed and convex conditional production set $Y_{k} \subset \mathbb{R}^{G}$ whose members are vectors with components that measure the net outputs per head of world population. In addition, $y_{k g}=0$ whenever $g \notin G_{k}$ and $y_{k} \in Y_{k}$ - i.e., $Y_{k} \subset \mathbb{R}^{G_{k}} \times\{0\}$. Finally, there is free disposal of nation $k$ 's tradable commodities in the sense that, if $y_{k} \in Y_{k}$ and $\tilde{y}_{k} \leqq y_{k}$ with $\tilde{y}_{k} \in \mathbb{R}^{G_{k}} \times\{0\}$, then $\tilde{y}_{k} \in Y_{k}$.

We allow that $0 \notin Y_{k}$ because of the need to produce the status quo public good vector in each nation.

\subsection{Feasible Allocations and the Status Quo}

An allocation $\left(\mathbf{x}^{I}, \mathbf{m}^{I}, \mathbf{y}^{K}\right)$ is a jointly measurable function $i \mapsto\left(x^{i}, m^{i}\right) \in$ $\mathbb{R}^{G} \times \mathbb{R}^{E D K}$ specifying both each individual's net trade vector and personal history, together with an international profile of net output vectors $\mathbf{y}^{K}=$ $\left\langle y^{k}\right\rangle_{k \in K} \in \mathbf{Y}^{K}=\prod_{k \in K} Y^{k}$. The allocation $\left(\mathbf{x}^{I}, \mathbf{m}^{I}, \mathbf{y}^{K}\right)$ is feasible if:

(i) $\left(x^{i}, m^{i}\right) \in X^{i}$ a.e. in $I$;

(ii) $y_{k} \in Y_{k}$ for all $k \in K$;

(iii) $\int_{I} x^{i} d \nu=\sum_{k \in K} y_{k}$. 
Condition (iii) requires the average net demand vector of all consumers to equal the aggregate net output of producers per head of world population.

The combined gains from free trade and migration will accrue from an allocation that is Pareto superior to a prespecified status quo feasible allocation $\left(\overline{\mathbf{x}}^{I}, \overline{\mathbf{m}}^{I}, \overline{\mathbf{y}}^{K}\right)$. Let $\overline{\mathbf{m}}^{I}$ denote the status quo distribution of personal histories in the world population. The corresponding congestion profile is $\bar{\mu}^{E D K}=\int_{I} \bar{m}^{i} d \nu$.

Relative to the status quo, for each $i \in I$, let

$$
\bar{X}^{i}:=\left\{\left(x^{i}, m^{i}\right) \in X^{i} \mid\left(x^{i}, m^{i}\right) \succsim^{i}\left(\bar{x}^{i}, \bar{m}^{i}\right)\right\}
$$

denote the set of $i$ 's allocations that are weakly preferred to the status quo. Assume that:

(A.5) For each $i \in I$, the set $\bar{X}^{i}$ has a lower bound $\underline{x}^{i} \in \mathbb{R}^{G}$ such that $\left(x^{i}, m^{i}\right) \in \bar{X}^{i}$ implies $x^{i} \geqq \underline{x}^{i}$; also, the mapping $i \mapsto \underline{x}^{i}$ is integrable with a mean lower bound $\underline{x}:=\int_{I} \underline{x}^{i} d \nu$ that is a finite vector in $\mathbb{R}^{G}$.

\subsection{Bounded Production Sets}

By (A.5), any feasible allocation $\left(\mathbf{x}^{I}, \mathbf{m}^{I}, \mathbf{y}^{K}\right)$ with $\left(x^{i}, m^{i}\right) \in \bar{X}^{i}$ for a.e. $i \in I$ must satisfy $\underline{x} \leqq \int_{I} x^{i} d \nu=\sum_{k \in K} y_{k}$. Define the set

$$
\mathbf{Y}^{K}(\underline{x}):=\left\{\mathbf{y}^{K} \in \mathbf{Y}^{K} \mid \sum_{k \in K} y_{k} \geqq \underline{x}\right\}
$$

of feasible international production allocations that this inequality. Assume that:

(A.6) The set $\mathbf{Y}^{K}(\underline{x})$ is bounded.

Thus, if aggregate global inputs are enough to allow an allocation that is Pareto non-inferior to the status quo, there are bounds on the outputs in each separate country, as well as in the international economy as a whole. 
Following the idea pioneered by Arrow and Debreu (1954), (A.6) implies there are compact sets $C_{k} \subset \mathbb{R}^{G}$ for each $k \in K$ which are large enough for the Cartesian product $\prod_{k \in K} C_{k}$ to contain $\mathbf{Y}^{K}(\underline{x})$ within its interior. Hence, any feasible allocation $\left(\mathbf{x}^{I}, \mathbf{m}^{I}, \mathbf{y}^{K}\right)$ with $\left(x^{i}, m^{i}\right) \in \bar{X}^{i}$ for a.e. $i \in I$ must satisfy $y_{k} \in \hat{Y}_{k}:=Y_{k} \cap C_{k}$ for all $k \in K$.

\subsection{Profit Maximization}

The domain of possible commodity price vectors is allowed to be the whole of $\mathbb{R}_{+}^{G}$. Later, Section 8 will discuss when there exists a non-zero equilibrium commodity price vector.

Assume that producers in each nation $k \in K$ maximize profits taking as given the price vector $p \geqq 0$, the fixed vector of public goods they have to supply, and the fixed national congestion profile $\bar{\mu}_{k}^{E D}$. For each nation $k \in K$ and price vector $p \geqq 0$, because each $\hat{Y}_{k}$ is compact, we can define

$$
\left.\begin{array}{rl}
\eta_{k}(p) & :=\arg \\
\pi_{k}(p) & :=
\end{array}\right\} \max _{y_{k}}\left\{p y_{k} \mid y_{k} \in \hat{Y}_{k}\right\}
$$

as the (non-empty) set of profit maximizing aggregate net output vectors and the associated aggregate profit per head of world population. Note that $\pi_{k}(p)$ may be negative because of the cost of producing public goods in nation $k$. Note too that, as Arrow and Debreu (1954) argue, any feasible allocation $\left(\mathbf{x}^{I}, \mathbf{m}^{I}, \mathbf{y}^{K}\right)$ with $\left(x^{i}, m^{i}\right) \in \bar{X}^{i}$ for a.e. $i \in I$ and $y_{k} \in \eta_{k}(p)$ for any $k \in K$ must satisfy $y_{k} \in \arg \max _{y_{k}}\left\{p y_{k} \mid y_{k} \in Y_{k}\right\}$.

\subsection{Residence Charges}

In our model of the world economy, migration creates externalities by adding to (or reducing) the congestion cost of providing public goods in both the 
source and destination localities. Given any fixed congestion profile $\mu^{E D K}$, a constrained Pareto efficient allocation of these externalities can be ensured by instituting a suitable Pigouvian tax scheme. The allocation is only constrained Pareto efficient because nothing is done to choose appropriate national public environments and congestion levels.

The Pigouvian tax scheme requires levying a net congestion or residence charge $t_{k}(e, d)$ on each consumer of characteristic $e$ living in country $k$ at date-event $d$. Then consumers choose their own personal histories, as well as their net trade vectors, subject to a budget constraint that reflects the appropriate residence charges for living in each location in each date-event.

Let $t \in \mathbb{R}^{E D K}$ denote the vector $\left\langle t_{k}(e, d)\right\rangle_{(e, d, k) \in E \times D \times K}$ of all residence charges. Each nation $k \in K$ is assumed to set its own residence charges $t_{k}^{E D}=\left\langle t_{k}(e, d)\right\rangle_{(e, d) \in E \times D}$ in order to clear markets with its congestion profile frozen at the status quo level $\bar{\mu}_{k}^{E D}$.

An extended price vector is a non-zero pair $(p, t) \in\left(\mathbb{R}_{+}^{G} \times \mathbb{R}^{E D K}\right) \backslash\{(0,0)\}$.

\subsection{Constrained Allocations}

Given the status quo congestion profile $\bar{\mu}^{E D K}$, define its support

$$
S:=\left\{(e, d, k) \in E \times D \times K \mid \bar{\mu}_{k}(e, d)>0\right\} .
$$

In order to maintain the status quo congestion profile, agents must be dissuaded from choosing any personal history outside the set

$$
M^{S}:=\left\{m \in M \mid m_{k}(e, d)=1 \Longrightarrow(e, d, k) \in S\right\} .
$$

In Section 7.4 below, we will show how this can be achieved by setting high enough residence charges $t_{k}(e, d)$ for all $(e, d, k) \notin S$. 
Furthermore, note that the status quo for agent $i$ satisfies $\bar{m}^{i} \notin M^{S}$ unless $\bar{m}_{k}^{i}(e, d)=1$ for some $(e, d, k) \in(E \times D \times K) \backslash S$ - i.e., for some $(e, d, k)$ such that $\bar{\mu}_{k}(e, d)=0$. Of course, this can happen only for a null set. It follows that $\bar{m}^{i} \in M^{S}$ a.e. in $I$. From now on, we disregard those $i \in I$ for whom $\bar{m}^{i} \notin M^{S}$. Also, define each consumer $i$ 's constrained set as

$$
\bar{X}^{i S}:=\left\{\left(x^{i}, m^{i}\right) \in \bar{X}^{i} \mid m^{i} \in M^{S}\right\}
$$

\section{Sagacious Wealth Distribution Rules}

\subsection{Wealth Distribution Rules}

A wealth distribution rule $\mathbf{w}^{I}(p, t)$ specifies each consumer $i$ 's wealth level $w^{i}$ as a function of the extended price vector $(p, t)$, and so determines each consumer's budget constraint $p x^{i}+t m^{i} \leq w^{i}(p, t)$.

Budget feasibility for the world as a whole requires that

$$
\int_{I} w^{i}(p, t) d \nu=\sum_{k \in K} \pi_{k}(p)+t \bar{\mu}^{E D K}
$$

The last term arises because the revenue from residence charges can either be distributed to consumers directly, or else used to reduce the losses of firms which provide public goods and so lower the taxes consumers must pay to subsidize these firms' losses. International transfers are allowed; see Hammond and Sempere (1995) for a discussion of when they can be avoided in the absence of migration.

\subsection{The Normalized Price Domain}

In each date-event $d \in D$, every individual $i \in I$ has to choose precisely one $(k, e) \in K \times E$ such that $m_{k}^{i}(e, d)=1$. This implies that only the algebraic 
differences $t_{k}(e, d)-t_{k^{\prime}}\left(e^{\prime}, d\right)$ between the residence charges in different locations $k, k^{\prime}$ for different kinds of household $e, e^{\prime}$ in each date-event $d$ are relevant to the left-hand side $p x^{i}+\sum_{d \in D} t_{k}(e, d) m_{k}(e, d)$ of the budget constraint $p x^{i}+t m^{i} \leq w^{i}(p, t)$. The absolute values of $t_{k}(e, d)$ are unimportant, except insofar as they amount to uniform head taxes (or subsidies) affecting everybody in the world, regardless of location $k$ and characteristic $e$. For this reason, we can always normalize prices by subtracting the minimum residence charge, so that in every date-event $d \in D$ the cheapest location has a normalized residence charge equal to zero.

Thus, let $T^{S}$ denote the set of vectors $t^{S} \in \mathbb{R}_{+}^{S}$ of residence charges satisfying $\min _{(e, k)}\left\{t_{k}(e, d) \mid(e, d, k) \in S\right\}=0$ for all $d \in D$. Then define

$$
\Delta^{S}:=\left\{\left(p, t^{S}\right) \in \mathbb{R}_{+}^{G} \times T^{S} \mid \sum_{g \in G} p_{g}+\sum_{(e, d, k) \in S} t_{k}(e, d)=1\right\}
$$

as the set of normalized price systems. Note that whenever $\left(p, t^{S}\right) \in \Delta^{S}$ one has $\sum_{g \in G} p_{g} \leq 1$ and $t^{S} m \geq 0$ for all $m \in M^{S}$.

\subsection{Sagacious Rules}

Given any extended price vector $(p, t) \in \mathbb{R}_{+}^{G} \times \mathbb{R}^{E D K}$, define

$$
\bar{e}^{i S}(p, t):=\inf _{x^{i}, m^{i}}\left\{p x^{i}+t m^{i} \mid\left(x^{i}, m^{i}\right) \in \bar{X}^{i S}\right\}
$$

This is the infimum wealth consumer $i$ needs to sustain the status quo standard of living, subject to the constraint $m^{i} \in M^{S}$. Note how (A.5) implies that $\bar{e}^{i S}(p, t)>-\infty$.

DeFINITION: The wealth distribution rule $\mathbf{w}^{I}(p, t)$ is sagacious if, for all $p \geqq 0$ and $t \in \mathbb{R}^{E D K}$, it satisfies (10) (i.e., it is budget feasible) and:

(i) whenever $\tilde{t} \in \mathbb{R}^{E D K}$ satisfies $\tilde{t}_{k}(e, d)=t_{k}(e, d)$ for all $(e, d, k) \in S$, then $w^{i}(p, t)=w^{i}(p, \tilde{t})$ for all $i \in I$; 
(ii) $w^{i}(p, t) \geq \bar{e}^{i S}(p, t)$ a.e. in $I$;

(iii) $\int_{I} \bar{e}^{i S}(p, t) d \nu<\sum_{k \in K} \pi_{k}(p)+t \bar{\mu}^{E D K}$ implies that $w^{i}(p, t)>\bar{e}^{i S}(p, t)$ a.e. in $I$.

Here part (i) reflects the idea that only the prices of residence permits for triples $(e, d, k) \in S$ are relevant for the equilibrium whose existence we wish to demonstrate. So the wealth distribution rule should not depend on the prices of other residence permits. Parts (ii) and (iii) are obvious adaptations of Grandmont and McFadden's (1972) original definition.

Note how expenditure minimization implies that $\bar{e}^{i S}(p, t) \leq p \bar{x}^{i}+t \bar{m}^{i}$ a.e. in $I$, whereas profit maximization implies $\pi_{k}(p) \geq p \bar{y}_{k}$ for all $k \in K$. Together with feasibility of the status quo allocation, these imply that

$$
\begin{aligned}
\int_{I} \bar{e}^{i S}(p, t) d \nu \leq \int_{I}\left(p \bar{x}^{i}+t \bar{m}^{i}\right) d \nu & =\sum_{k \in K} p \bar{y}_{k}+t \bar{\mu}^{E D K} \\
& \leq \sum_{k \in K} \pi_{k}(p)+t \bar{\mu}^{E D K}
\end{aligned}
$$

for all $p \geqq 0$ and $t \in \mathbb{R}^{E D K}$. It follows that (ii) is always possible. So are (i) and (iii) if, for instance, we choose

$$
w^{i}(p, t) \equiv \bar{e}^{i S}(p, t)+\theta^{i}\left[\sum_{k \in K} \pi_{k}(p)+t \bar{\mu}^{E D K}-\int_{I} \bar{e}^{i S}(p, t) d \nu\right]
$$

for positive constants $\theta^{i}$ (all $i \in I$ ) such that the function $i \mapsto \theta^{i}$ is integrable, with $\int_{I} \theta^{i} d \nu=1$. The key Lemma 1 in Hammond and Sempere (2006) confirms that such a rule is integrable w.r.t. $i$, and continuous w.r.t. $(p, t)$.

After this discussion, an obvious assumption is that:

(A.7) The wealth distribution rule $\mathbf{w}^{I}(p, t)$ is integrable w.r.t. $i$, continuous w.r.t. $(p, t)$, and sagacious. 


\section{Constrained Compensated Equilibrium}

\subsection{Conditional Walrasian Equilibrium}

Given the frozen levels of public goods and congestion profile, as well as the wealth distribution rule $\mathbf{w}^{I}(p, t)$, and the particular extended price vector $(p, t) \in \mathbb{R}_{+}^{G} \times \mathbb{R}^{E D K}$, define each consumer $i$ 's conditional budget set as

$$
B^{i}(p, t):=\left\{\left(x^{i}, m^{i}\right) \in X^{i} \mid p x^{i}+t m^{i} \leq w^{i}(p, t)\right\} .
$$

Then consumer $i$ 's conditional Walrasian or uncompensated demand set $\gamma^{i}(p, t)$ is defined as

$$
\left\{\left(\hat{x}^{i}, \hat{m}^{i}\right) \in B^{i}(p, t) \mid\left(x^{i}, m^{i}\right) \succ^{i}\left(\hat{x}^{i}, \hat{m}^{i}\right) \Longrightarrow p x^{i}+t m^{i}>w^{i}(p, t)\right\} .
$$

It is easy to see how assumptions (A.1), (A.2), and (A.7) together imply that $\gamma^{i}(p, t) \subset \bar{X}^{i}$ a.e. in $I$. In fact, the constraint $\left(x^{i}, m^{i}\right) \in \bar{X}^{i}$ cannot bind when the wealth distribution rule is sagacious and so $w^{i}(p, t) \geq \bar{e}^{i S}(p, t)$.

Given both fixed quantities of public goods and the fixed congestion profile $\bar{\mu}^{E D K}=\int_{I} \bar{m}^{I} d \nu$, a conditional Walrasian equilibrium is a feasible allocation $\left(\hat{\mathbf{x}}^{I}, \hat{\mathbf{m}}^{I}, \hat{\mathbf{y}}^{K}\right)$, together with an extended price vector $(\hat{p}, \hat{t}) \in \mathbb{R}_{+}^{G} \times$ $\mathbb{R}^{E D K}$, such that:

$$
\begin{aligned}
\text { (i) } & \int_{I} \hat{m}^{i} d \nu=\bar{\mu}^{E D K} \\
\text { (ii) } & \hat{y}_{k} \in \eta_{k}(\hat{p}) \text { for all } k \in K \\
\text { (iii) } & \left(\hat{x}^{i}, \hat{m}^{i}\right) \in \gamma^{i}(\hat{p}, \hat{t}) \text { a.e. in } I .
\end{aligned}
$$

Using (4) and (9), we also define the constrained budget set

$$
\bar{B}^{i S}(p, t):=\left\{\left(x^{i}, m^{i}\right) \in \bar{X}^{i S} \mid p x^{i}+t m^{i} \leq w^{i}(p, t)\right\} .
$$

Consumer $i$ 's constrained compensated demand set $\bar{\gamma}_{C}^{i S}(p, t)$ is defined as

$$
\left\{\left(\hat{x}^{i}, \hat{m}^{i}\right) \in \bar{B}^{i S}(p, t) \mid\left(x^{i}, m^{i}\right) \succsim^{i}\left(\hat{x}^{i}, \hat{m}^{i}\right) \Longrightarrow p x^{i}+t m^{i} \geq w^{i}(p, t)\right\} .
$$


A constrained compensated equilibrium is like a conditional Walrasian equilibrium in that it satisfies parts (i) and (ii) of the definition, but only the modified version of part (iii) requiring that $\left(\hat{x}^{i}, \hat{m}^{i}\right) \in \bar{\gamma}_{C}^{i S}(\hat{p}, \hat{t})$ a.e. in $I$. Of course, this implies that $\left(\hat{x}^{i}, \hat{m}^{i}\right) \succsim^{i}\left(\bar{x}^{i}, \bar{m}^{i}\right)$ a.e. in $I$.

\subsection{A First Existence Result}

Our first result is that, given the fixed congestion levels $\bar{\mu}^{E D K}$, there exists a constrained compensated equilibrium.

Lemma 1: Suppose assumptions (A.1)-(A.7) are all satisfied. Then there is a constrained compensated equilibrium $\left(\hat{\mathbf{x}}^{I}, \hat{\mathbf{m}}^{I}, \hat{\mathbf{y}}^{K}, \hat{p}, \hat{t}\right)$.

Proof: We apply Lemma 2 in Hammond and Sempere (2006), to the commodity space $\mathbb{R}^{G} \times \mathbb{R}^{S}$. It demonstrates existence of a constrained compensated equilibrium with free disposal. That is, there must exist $\left(\tilde{\mathbf{x}}^{I}, \hat{\mathbf{m}}^{I}, \tilde{\mathbf{y}}^{K}, \hat{p}, \hat{t}\right)$ satisfying $\left(\tilde{x}^{i}, \hat{m}^{i}\right) \in \bar{\gamma}_{C}^{i S}(\hat{p}, \hat{t})$ for a.e. $i \in I$, as well as $\tilde{y}_{k} \in \eta_{k}(\hat{p})$ for all $k \in K$, with $\int_{I} \tilde{x}^{i} d \nu \leqq \sum_{k \in K} \tilde{y}_{k}$ and

$$
\int_{I} \hat{m}_{k}^{i}(e, d) d \nu \leq \bar{\mu}_{k}(e, d)
$$

for all $(e, d, k) \in S$. But by definition (7), for all $(e, d, k) \notin S$ one has $\bar{\mu}_{k}(e, d)=0=\hat{m}_{k}^{i}(e, d)$ a.e. in $I$. Also, definition (1) guarantees that

$$
\sum_{(k, e) \in K \times E} \hat{m}_{k}^{i}(e, d)=1=\sum_{(k, e) \in K \times E} \bar{\mu}_{k}(e, d)
$$

for all $i \in I$ and $D \in D$, implying that

$$
\sum_{(k, e) \in K \times E} \int_{I} \hat{m}_{k}^{i}(e, d) d \nu=1=\sum_{(k, e) \in K \times E} \bar{\mu}_{k}(e, d) .
$$

Combining this with (17), it follows that $\int_{I} \hat{m}^{i} d \nu=\bar{\mu}^{E D K}$. 
Next, arguing as in the proof of Lemma 3 in Hammond and Sempere (2006), the free disposal properties implied by (A.1) and (A.2), or alternatively by (A.6), guarantee the existence of an alternative pair $\left(\hat{\mathbf{x}}^{I}, \hat{\mathbf{y}}^{K}\right)$ with $\hat{x}^{i} \geqq \tilde{x}^{i}$ for almost all $i \in I$ and $\hat{y}_{k} \leqq \tilde{y}_{k}$ for all $k \in K$ satisfying $\hat{p} \hat{x}^{i}=\hat{p} \tilde{x}^{i}$ a.e. in $I$, as well as $\hat{p} \hat{y}_{k}=\hat{p} \tilde{y}_{k}$ for all $k \in K$, with $\int_{I} \hat{x}^{i} d \nu=\sum_{k \in K} \hat{y}_{k}$. Then $\left(\hat{\mathbf{x}}^{I}, \hat{\mathbf{m}}^{I}, \hat{\mathbf{y}}^{K}, \hat{p}, \hat{t}\right)$ is the required constrained compensated equilibrium.

\section{Bounding the Prices of Residence Permits}

\subsection{First Example with Zero Commodity Prices}

For the following two examples, the only possible extended price vectors in constrained compensated equilibrium may have zero prices for all physical commodities in the economy, with non-zero prices only for residence permits. Because of non-satiation in commodities, such an extended price vector can never be a Walrasian equilibrium. This is true even though the examples satisfy assumptions (A.1)-(A.6).

Suppose there is a single good, so $\# G=1$, and just one date-event, so $D=\{0\}$. Assume the set $K$ consists of two nations, labelled $A$ and $B$. Finally, assume there is only one crowding type, which can therefore be ignored. Thus, each $i \in I$ has a personal history $k^{i} \in K=\{A, B\}$.

Each consumer $i \in I$ is assumed to have a conditional feasible set $X^{i}(A)=X^{i}(B)$ which, for some real lower bound $\underline{x}^{i}<0$, is equal to the closed half-line $\left[\underline{x}^{i}, \infty\right) \subset \mathbb{R}$, independent of the choice of residence.

In the first example we assume that the lower bounds $\underline{x}^{i}$ of all individuals $i \in I$ are distributed with a positive continuous density over the whole of the closed half-line $(-\infty,-1] \subset \mathbb{R}$. For example, the distribution could be truncated normal. Finally, assume each consumer $i \in I$ has preferences on 
the space $\left[\underline{x}^{i}, \infty\right) \times\{A, B\}$ of net trade/country pairs which are represented by the utility function $u^{i}(x, k)$ defined for all $x \geqq \underline{x}^{i}$ by

$$
u^{i}(x, A):=x-2 \underline{x}^{i} \quad \text { and } \quad u^{i}(x, B):=x .
$$

Suppose the status quo allocation is autarkic, with all consumers residing in $B$. Hence, $\left(\bar{x}^{i}, \bar{k}^{i}\right)=(0, B)$ for all $i \in I$, and $S=\{B\}$. Then the only feasible allocation with the same congestion profile and with $\left(\hat{x}^{i}, \hat{k}^{i}\right) \succsim^{i}$ $(0, B)$ for a.e. $i \in I$ must have $\hat{k}^{i}=B$ and $\hat{x}^{i} \geq 0$. But then feasibility implies $\hat{x}^{i}=0$ for a.e. $i \in I$. Hence, the only such allocation combines autarky with immobility.

Let $\mathbf{w}^{I}(p, t)$ be any wealth distribution rule satisfying (A.7). In order for $\left(p, t_{A}, t_{B}\right)$ to be a consstrained compensated equilibrium price vector, for a.e. $i \in I$ one must have $p x^{i} \geq p \hat{x}^{i}=0$ whenever $x^{i} \geq \hat{x}^{i}=0$. So $p \geq 0$.

Furthermore, for a.e. $i \in I$, whenever $x^{i} \geq 2 \underline{x}^{i}$ one has $\left(x^{i}, A\right) \succsim^{i}(0, B)$ and so $p x^{i}+t_{A} \geq t_{B}$. In particular, $t_{A} \geq t_{B}$ when $x^{i}=0$ and $2 p \underline{x}^{i}+t_{A} \geq t_{B}$ when $x^{i}=2 \underline{x}^{i}$. Our assumptions imply, however, that no matter how large the real positive constant $c$ may be, there exists a non-null set $I_{c}$ of individuals $i \in I$ for whom $\underline{x}^{i} \leq-c$ and so $p c \leq 2\left(t_{A}-t_{B}\right)$. This is only possible if $p \leq 0$; therefore $p=0 .^{3}$

\subsection{Second Example with Zero Commodity Prices}

The second example shares many features in common with the first. There are two types of consumers, however, each with a common distribution of the lower bounds $\underline{x}^{i}$ represented by a continuous density function restricted to the interval $[-2,-1]$. Consumers of congestion type $a$ have preferences

\footnotetext{
${ }^{3}$ This example also satisfies assumptions (A.8)-(A.11) set out below. In particular, (A.9) is trivially satisfied because $S=\{B\}$ and so $\Delta^{S}$ collapses to the single point $\left\{\bar{\mu}^{S}\right\}$.
} 
represented by a utility function $u^{i}(x, k)$ with

$$
u^{i}(x, A):=x \quad \text { and } \quad u^{i}(x, B):=x+3,
$$

whereas those of congestion type $b$ have preferences represented by

$$
u^{i}(x, A):=x \quad \text { and } \quad u^{i}(x, B):=x .
$$

The status quo is autarky with all type $a$ consumers living in $A$, but positive proportions of type $b$ consumers living in each country.

As in the first example, the only feasible allocation with the same congestion profile and with $\left(\hat{x}^{i}, \hat{k}^{i}\right) \succsim^{i}(0, B)$ for a.e. $i \in I$ must combine autarky with immobility. Also, again as in the first example, given any wealth distribution rule $\mathbf{w}^{I}(p, t)$ satisfying (A.7), in order for $\left(p, t_{A}, t_{B}\right)$ to be a constrained compensated equilibrium price vector, one must have $p \geq 0$.

For type $a$ consumers, compensated equilibrium requires $t^{B}+p x^{i} \geq t^{A}$ for all $x^{i} \geq-2$, so $t^{B}-2 p \geq t^{A}$. For type $b$ consumers, compensated equilibrium requires $t^{A}+p x^{i} \geq t^{B}$ for all $x^{i} \geq 0$, so $t^{A} \geq t^{B}$. Hence $t^{B}-2 p \geq t^{A} \geq t^{B}$, which is only possible if $p \leq 0$; therefore $p=0 .{ }^{4}$

\subsection{Boundedly Sagacious Rules}

The crucial feature of the example in Section 7.1 is the lack of any uniform upper bound on the amount individuals are willing to pay for being allowed to move from $B$ to $A$. An extra assumption will bound what consumers can afford to pay for relevant changes in personal history.

Let $1^{G}$ denote the vector $(1,1, \ldots, 1) \in \mathbb{R}^{G}$. The sagacious wealth distribution rule $\mathbf{w}^{I}(p, t)$ is said to be boundedly sagacious if there exists a uniform scalar bound $\alpha \in \mathbb{R}_{+}$such that the status quo allocation

\footnotetext{
${ }^{4}$ This second example also satisfies assumptions (A.7*) and (A.9)-(A.11) set out below.
} 
$\left(\overline{\mathbf{x}}^{I}, \overline{\mathbf{m}}^{I}, \overline{\mathbf{y}}^{K}\right)$, and the lower bounds $\underline{x}^{i}(i \in I)$ of (A.5), together satisfy $w^{i}(p, t) \leq p\left(\underline{x}^{i}+\alpha 1^{G}\right)+t \bar{m}^{i}$ for a.e. $i \in I$, and for all $(p, t) \in \mathbb{R}_{+}^{G} \times \mathbb{R}^{E D K}$.

After this definition, we strengthen (A.7) by assuming that:

$\left(\right.$ A. $\left.7^{*}\right)$ The wealth distribution rule $\mathbf{w}^{I}(p, t)$ is integrable w.r.t. $i$, continuous w.r.t. $(p, t)$, and boundedly sagacious.

A wealth distribution rule $\mathbf{w}^{I}(p, t)$ satisfying $\left(\right.$ A. $\left.7^{*}\right)$ exists provided that $\bar{e}^{i S}(p, t) \leq p\left(\underline{x}^{i}+\alpha 1^{G}\right)+t \bar{m}^{i}$. But $\bar{e}^{i S}(p, t) \leq p \bar{x}^{i}+t \bar{m}^{i}$ and $p \geqq 0$. So an obvious sufficient condition allowing $\left(\right.$ A. $\left.7^{*}\right)$ to be satisfied is that the status quo net trade vectors satisfy $\bar{x}^{i} \leqq \underline{x}^{i}+\alpha 1^{G}$ for a.e. $i \in I$, with $\alpha$ independent of $i$. This assumption is actually quite reasonable, but unnecessarily strong.

\subsection{The Restricted Price Domain}

In Section 4.6 it was claimed that setting residence charges sufficiently high would dissuade consumers from choosing any personal history outside the set $M^{S}$. Given that $\bar{m}^{i} \in M^{S}$ for a.e. $i \in I$, this claim can now be justified by the following result:

Lemma 2: Assume (A.1)-(A.6) and (A.7*) are satisfied, and $(p, t) \in \Delta^{S}$ with

$$
t_{k}(e, d)>\alpha+\max _{m}\left\{t m \mid m \in M^{S}\right\} \text { for all }(e, d, k) \notin S .
$$

Then any $i \in I$ with $\bar{m}^{i} \in M^{S}$ has $m^{i} \in M^{S}$ for all $\left(x^{i}, m^{i}\right) \in B^{i}(p, t)$, as well as $\bar{X}^{i S}=\bar{X}^{i}$ and $\bar{B}^{i S}(p, t)=B^{i}(p, t) \cap \bar{X}^{i}$.

Proof: Consider any $\left(x^{i}, m^{i}\right) \in \bar{B}^{i S}(p, t)$. By (A.5) one has $\left(x^{i}, m^{i}\right) \in \bar{X}^{i}$ and so $x^{i} \geqq \underline{x}^{i}$. But $p \geqq 0$, implying that $p x^{i}+t m^{i} \geq p \underline{x}^{i}+t m^{i}$. Then $\left(\mathrm{A} .7^{*}\right)$ implies that $p \underline{x}^{i}+t m^{i} \leq w^{i}(p, t) \leq p\left(\underline{x}^{i}+\alpha 1^{G}\right)+t \bar{m}^{i}$ and so $t\left(m^{i}-\bar{m}^{i}\right) \leq p \alpha 1^{G} \leq \alpha$, given that $p 1^{G} \leq 1$ for all $(p, t) \in \Delta^{S}$. 
Consider any $(e, d, k) \notin S$. Because $\bar{m}^{i} \in M^{S}$, the hypothesis of the lemma implies that $t m^{i} \leq p \alpha 1^{G}+t \bar{m}^{i}<t_{k}(e, d)$. Because $t \geqq 0$ and $m_{k}^{i}(e, d) \in\{0,1\}$ for all $(e, d, k) \in E \times D \times K$, it follows that $m_{k}^{i}(e, d)=0$ for all $(e, d, k) \notin S$ - i.e., that $m^{i} \in M^{S}$.

The rest of the proof is immediate from definitions (8) and (9).

For the conclusion of Lemma 2 to hold, it is enough to make the residence charges $t_{k}(e, d)$ sufficiently high for all $(e, d, k) \notin S$. Indeed, consider a constrained compensated equilibrium price vector $\left(\hat{p}, \hat{t}^{S}\right)$ that clears markets for commodities $g \in G$ and for residence permits $(e, d, k) \in S$. Then Lemma 2 states that, given any normalized price system $(p, t) \in \Delta^{S}$ as in (11), residence charges for $(e, d, k) \notin S$ can be set high enough to make the constraints $m^{i} \in M^{S}$ self-enforcing. Accordingly, from now on these other residence charges can be effectively ignored. We refer simply to a "compensated equilibrium", without mentioning the constraints $m^{i} \in M^{S}$. Lemmas 1 and 2 assure us that such an equilibrium exists.

\subsection{A Desirability Assumption}

The example in Section 7.2 shows that our assumptions so far still do not exclude a zero commodity price vector in compensated equilibrium. To ensure that at least one divisible commodity has a positive price when some commodities are indivisible, Broome (1972) introduced the assumption that divisible goods were overridingly desirable — see also Mas-Colell (1977). In our model, for all $i \in I$, given any $\left(x^{i}, m^{i}\right) \in X^{i}$ and $\tilde{m}^{i} \in M$, this would require the existence of an $\tilde{x}^{i} \in X^{i}\left(\tilde{m}^{i}\right) \operatorname{such}$ that $\left(\tilde{x}^{i}, \tilde{m}^{i}\right) \succ^{i}\left(x^{i}, m^{i}\right)$.

Yet some personal histories may be very distasteful to some individuals. Or they may involve excessive migration, thus making them very expensive 
and inconvenient, if not entirely impossible. Accordingly, we define the set

$$
\bar{M}^{i S}:=\left\{m^{i} \in M^{S} \mid \exists x^{i} \in \mathbb{R}^{G}:\left(x^{i}, m^{i}\right) \in \bar{X}^{i}\right\}
$$

of $i$ 's personal histories in $M^{S}$ which, when combined with a sufficiently desirable commodity bundle, are non-inferior to the status quo. Now we can state the following restricted version of overriding desirability:

(A.8) For almost all $i \in I$, whenever $\left(x^{i}, m^{i}\right) \in \bar{X}^{i}$ and $\tilde{m}^{i} \in \bar{M}^{i S}$, there exists $\tilde{x}^{i} \in X^{i}\left(\tilde{m}^{i}\right)$ such that $\left(\tilde{x}^{i}, \tilde{m}^{i}\right) \succsim^{i}\left(x^{i}, m^{i}\right)$.

\subsection{Interiority}

The final assumption in this section is an interiority condition of a kind that is familiar in general equilibrium existence proofs.

First, for each $d \in D$, let $S(d):=\{(e, k) \in E \times K \mid(e, d, k) \in S\}$ denote the section of $S$ for the given date-event $d$. Let $\Delta^{S(d)}$ denote the unit simplex in the associated Euclidean space $\mathbb{R}^{S(d)}$. Thus,

$$
\Delta^{S(d)}:=\left\{\left\langle\mu_{k}(e, d)\right\rangle_{(e, k) \in S(d)} \in \mathbb{R}_{+}^{S(d)} \mid \sum_{(e, k) \in S(d)} \mu_{k}(e, d)=1\right\} .
$$

Of course, the Cartesian product $\prod_{d \in D} \Delta^{S(d)}$ is a subset of the non-negative orthant $\mathbb{R}_{+}^{S}$. We assume:

(A.9) The point $\bar{\mu}^{S}$ lies in the interior of $\int_{I} \bar{M}^{i S} d \nu$ relative to $\prod_{d \in D} \Delta^{S(d)}$.

That is, for each $d \in D$ there must be an open set $U(d) \subset \mathbb{R}^{S(d)}$ with

$$
\bar{\mu}^{S} \in \prod_{d \in D}\left[U(d) \cap \Delta^{S(d)}\right] \subset \int_{I} \bar{M}^{i S} d \nu
$$

To illustrate this assumption, suppose some environmental disaster were to compel a small enough proportion of the population to change their personal histories from the status quo, while still remaining within the set $M^{S}$. 
Then (A.9) requires that, with sufficient resources and with some population exchanges from this modified status quo, one can still reach an allocation that is Pareto non-inferior to the original status quo.

\subsection{A Non-Zero Commodity Price Vector}

Lemma 3: Suppose assumptions (A.1)-(A.6), (A.7*) and (A.8)-(A.9) are satisfied. Then there is a compensated equilibrium $\left(\hat{\mathbf{x}}^{I}, \hat{\mathbf{m}}^{I}, \hat{\mathbf{y}}^{K}, \hat{p}, \hat{t}\right)$ which satisfies $\left(\hat{x}^{i}, \hat{m}^{i}\right) \in \bar{X}^{i}$ a.e. in $I$, with $\hat{p}>0$.

Proof: By Lemmas 1 and 2, there is a constrained compensated equilibrium $\left(\hat{\mathbf{x}}^{I}, \hat{\mathbf{m}}^{I}, \hat{\mathbf{y}}^{K}, \hat{p}, \hat{t}\right)$ satisfying $\left(\hat{x}^{i}, \hat{m}^{i}\right) \in \bar{X}^{i}$ a.e. in $I$. This is also a compensated equilibrium provided we make $\hat{t}$ satisfy (18). So we only have to show that $\hat{p}>0$.

Suppose $\hat{p}=0$ and consider any $\tilde{m}^{i} \in \bar{M}^{i S}$. Under assumption (A.8), there exists $\tilde{x}^{i} \in X^{i}\left(\tilde{m}^{i}\right)$ such that $\left(\tilde{x}^{i}, \tilde{m}^{i}\right) \succsim^{i}\left(\hat{x}^{i}, \hat{m}^{i}\right)$. Because $\hat{p}=0$, the definition of compensated equilibrium implies that $\hat{t} \tilde{m}^{i} \geq \hat{t} \hat{m}^{i}$. In particular, given that $\bar{m}^{i} \in \bar{M}^{i S}$, one has $\hat{t} \bar{m}^{i} \geq \hat{t} \hat{m}^{i}$ a.e. in $I$. But

$\bar{\mu}=\int_{I} \bar{m}^{i} d \nu=\int_{I} \hat{m}^{i} d \nu$ in the compensated equilibrium, implying that $\int_{I} \hat{t} \bar{m}^{i} d \nu=\int_{I} \hat{t} \hat{m}^{i} d \nu$, and so $\hat{t} \bar{m}^{i}=\hat{t} \hat{m}^{i}$ a.e. in $I$. It follows that $\hat{t} m^{i} \geq \hat{t} \bar{m}^{i}$ whenever $m^{i} \in \bar{M}^{i S}$, and so $\hat{t} \mu \geq \hat{t} \bar{\mu}$ whenever $\mu \in \int_{I} \bar{M}^{i S} d \nu$. This evidently contradicts (A.9).

Hence, by contraposition, if (A.1)-(A.6), (A.7*) and (A.8)-(A.9) are all satisfied, then only $\hat{p}>0$ is possible.

\section{Conditional Walrasian Equilibrium}

Our one remaining task is to show that, in our economy with fixed public goods, a fixed congestion profile, and a boundedly sagacious wealth distri- 
bution rule, there exists a conditional Walrasian equilibrium market clearing extended price vector which includes residence charges. To do this, we shall provide additional assumptions ensuring that the conditional compensated equilibrium found in Lemma 3 is also a conditional Walrasian equilibrium. The extra assumptions are required to overcome an additional complication due to boundary problems, as discussed in the next subsection.

\subsection{Convexity and the Cheaper Point Lemma}

By construction, a sagacious wealth distribution rule generates lump-sum transfers enabling each consumer to afford at least the status quo standard of living. And if $\int_{I} \bar{e}^{i S}(p, t) d \nu<\sum_{k \in K} \pi_{k}(p)+t \bar{\mu}^{E D K}$, then almost every consumer $i$ will have some cheaper point $\left(x^{i}, m^{i}\right)$ in the conditional feasible set $X^{i}$ that satisfies $p x^{i}+t m^{i}<w^{i}(p, t)$. However, in our model, this may not be enough to prevent a non-null set of individuals $i \in I$ from demanding some pair $\left(\hat{x}^{i}, \hat{m}^{i}\right)$ with a net trade vector $\hat{x}^{i}$ that is a cheapest point of the relevant conditional feasible set $X^{i}\left(\hat{m}^{i}\right)$ given the chosen personal history $\hat{m}^{i}$. This creates a boundary problem which could prevent the existence of conditional Walrasian equilibrium.

This problem will be resolved by making use of two additional assumptions, similar to those used in Hammond and Sempere (2006). First:

(A.10) For every $i \in I$ and $m^{i} \in M$, the set $X^{i}\left(m^{i}\right)$ is convex.

This convexity assumption enables us to use the following extension of the usual cheaper point lemma.

Lemma 4: Suppose that (A.1), (A.2) and (A.10) are satisfied. For any fixed pair $i \in I$ and $(p, t) \in \Delta^{S}$ satisfying (18), let $\left(\hat{x}^{i}, \hat{m}^{i}\right) \in \gamma_{C}^{i S}(p, t)$ be a

constrained compensated demand. Suppose too that, whenever $\left(x^{i}, m^{i}\right) \succ^{i}$ 
$\left(\hat{x}^{i}, \hat{m}^{i}\right)$, there exists a "conditional cheaper point" $\tilde{x}^{i} \in X^{i}\left(m^{i}\right)$ satisfying $p \tilde{x}^{i}+t m^{i}<w^{i}(p, t)$. Then $\left(\hat{x}^{i}, \hat{m}^{i}\right) \in \gamma^{i}(p, t)$.

Proof: The proof is virtually identical to that of Lemma 3 in Hammond and Sempere (2006); there is just extra notation because $m^{i}$ is included in the commodity space and $t$ in the price vector.

\subsection{Dispersion}

Next, we invoke an additional "dispersion" assumption similar to that used in Hammond and Sempere (2006), where it is further discussed and analysed. To state this assumption formally in the present framework, first define

$$
\underline{w}^{i}\left(p, t, m^{i}\right):=\min _{x^{i}}\left\{p x^{i}+t m^{i} \mid x^{i} \in X^{i}\left(m^{i}\right)\right\}
$$

as the minimum wealth needed by consumer $i$ at prices $(p, t) \in \Delta^{S}$ with $p>0$ in order to sustain the personal history $m^{i}$. If the minimum does not exist, we take $\underline{w}^{i}\left(p, t, m^{i}\right):=+\infty$. Under assumption (A.3), for each fixed $m \in M^{S}$ the mapping $i \mapsto \underline{w}^{i}(p, t, m)$ is measurable.

Recall definition (19) and then, for each $m \in M$, let

$$
\bar{M}:=\left\{m \in M^{S} \mid \nu\left(\left\{i \in I \mid m \in \bar{M}^{i S}\right\}\right)>0\right\}
$$

denote the set of personal histories which are non-inferior for a non-null set of individuals. Clearly $\bar{M}$ includes the support of the status quo distribution of personal histories $\bar{m}^{i}(i \in I)$, but it could be much more extensive.

Finally, for each $i \in I$ and each $(p, t) \in \Delta^{S}$ with $p>0$, define

$$
I^{*}(p, t):=\left\{i \in I \mid \exists m \in \bar{M}: w^{i}(p, t)=\underline{w}^{i}(p, t, m)\right\}
$$

as the set of individuals with a critical level of wealth that is just enough to afford one of the individual histories $m \in \bar{M}$. The dispersion assumption is: (A.11) For all $(p, t) \in \Delta^{S}$ with $p>0$, the set $I^{*}(p, t)$ has measure zero in $I$. 


\subsection{Existence of Conditional Walrasian Equilibrium}

It remains to show that there exists a conditional Walrasian equilibrium with gains from migration.

Proposition: Under assumptions (A.1)-(A.6), (A.7*), and (A.8)-(A.11), and given the fixed status quo congestion profile $\bar{\mu}^{E D K}=\int_{I} \bar{m}^{i} d \nu$, there exists a conditional Walrasian equilibrium $\left(\hat{\mathbf{x}}^{I}, \hat{\mathbf{m}}^{I}, \hat{\mathbf{y}}^{K}, \hat{p}, \hat{t}\right)$ with $\int_{I} \hat{m}^{i} d \nu=$

$\bar{\mu}^{E D K}$ and $\left(\hat{x}^{i}, \hat{m}^{i}\right) \succsim^{i}\left(\bar{x}^{i}, \bar{m}^{i}\right)$ a.e. in I. Also, except when the status quo is already a conditional Walrasian equilibrium, one has $\left(\hat{x}^{i}, \hat{m}^{i}\right) \succ^{i}\left(\bar{x}^{i}, \bar{m}^{i}\right)$ a.e. in $I$.

Proof: By Lemma 3, assumptions (A.1)-(A.6), (A.7*) and (A.8)-(A.9) together imply that there exists a conditional compensated equilibrium $\left(\hat{\mathbf{x}}^{I}, \hat{\mathbf{m}}^{I}, \hat{\mathbf{y}}^{K}, \hat{p}, \hat{t}\right)$ with $\hat{p}>0$ and $\left(\hat{x}^{i}, \hat{m}^{i}\right) \in \bar{X}^{i}$ a.e. in I. Using (A.10), (A.11), and Lemma 4, the proof of Hammond and Sempere (2006, Lemma 4) is easily adapted to show that the conditional compensated equilibrium is a conditional Walrasian equilibrium. And also that $\left(\hat{x}^{i}, \hat{m}^{i}\right) \succ^{i}\left(\bar{x}^{i}, \bar{m}^{i}\right)$ for a.e. $i \in I$ unless the status quo itself is a conditional Walrasian equilibrium.

\section{$9 \quad$ Final Remarks}

We have extended the results of Hammond and Sempere (2006) to an economy with public goods subject to congestion where all nations or localities keep their public goods and congestion profiles at the status quo levels. This still allows consumers to exchange places freely, mediated by a system of residence charges. If the world economy could move to an improved allocation of public goods together with congestion profiles, the combined gains 
to trade and migration (or changing places) would be augmented. Such further improvements, however, go beyond the scope of this paper.

\section{Acknowledgements}

Most of the acknowledgements in Hammond and Sempere (2006) apply here also, including the disclosure that we are migrants ourselves.

The choice of title is inspired in part by David Lodge's novel Changing Places. Nor was it an accident that Hammond presented earlier versions of the paper to seminars in the Economics Departments of the University of Birmingham ("Rummidge") and the University of California at Berkeley ("Euphoric State"). Our thanks to the members of those and numerous other seminar audiences.

\section{References}

Arrow, K.J. And G. Debreu (1954), "Existence of Equilibrium for a Competitive Economy," Econometrica 22, 265-290.

Broome, J. (1972), "Existence of Equilibrium in Economies with Indivisible Commodities," Journal of Economic Theory 5, 224-250.

Conley, J. And M.H. Wooders (1996), "Taste-Homogeneity of Optimal Jurisdictions in a Tiebout Economy with Crowding Types and Endogenous Educational Investment Choices," Ricerche Economiche 50, 367-387.

Conley, J. And M.H. Wooders (1997) "Equivalence of the Core and Competitive Equilibrium in a Tiebout Economy with Crowding Types," Journal of Urban Economics 41, 421-440.

Hammond P.J. And J. Sempere (1995), "Limits to the Potential Gains from Market Integration and Other Supply-Side Policies," Economic Journal 105, 1180-1204. 
Hammond, P.J. and J. Sempere (2006), "Gains from Trade versus Gains from Migration: What Makes Them So Different?" Journal of Public Economic Theory 8, 145-170.

Hildenbrand, W. (1974), Core and Equilibria of a Large Economy (Princeton: Princeton University Press).

Mas-Colell, A. (1977), "Indivisible Commodities and General Equilibrium Theory," Journal of Economic Theory 16, 443-456. 